\title{
A Cylindrical Surface Model to Rectify the Bound Document Image
}

\author{
Huaigu Cao, Xiaoqing Ding, Changsong Liu \\ Department of Electronic Engineering, Tsinghua University \\ State Key Laboratory of Intelligent Technology and Systems \\ Beijing, 100084, P. R. China \\ Email: caohg@ocrserv.ee.tsinghua.edu.cn
}

\begin{abstract}
This article proposes a novel approach on how to rectify the photo image of the bound document. The surface of the document is modeled by a cylindrical surface. By the geometry of camera image formation, the equations using the cue of directrixes to map the points on the surface in the 3-D scene to the points on the image plane are achieved. Baselines of the horizontal text line are extracted as projections of directrixes to estimate the bending extent of the surface, and then the images are rectified. The proposed method needs no auxiliary device. Experimental results are presented to demonstrate the feasibility and the application of the method.
\end{abstract}

\section{Introduction}

This article has been motivated by the advantage of using digital camera for document image capture. Compared with the scanner, the digital camera is quite easier to use, being able to capture images from any viewpoint. However, the conveniences of using digital camera are accompanied with some image quality problems. When one takes a photo of a document page, one may often find that the resulting image is warped. This is generally caused by the existence of bookbinding, which prevents the page from being flattened. One may imagine that, with a scanner, one can make the documents flat simply by pressing them intentionally on the glass board. An example of warping caused by bookbinding is shown in Figure 1. In this case, the process of document layout analysis and OCR will become complicated and unreliable, and it is hard to restore the position or the size of the characters accurately.

There have been a number of literatures concerning the rectification of this kind of warping. In [1], a laser projector is used to project a $2 \mathrm{D}$ light network on the surface of the document, and then two dimensional distortions of the surface are corrected with a two pass mesh warping proposed by [2]. This method needs additional device and calibration. The method introduced in [3] is to get the depth of each point in the image by some stereo vision method, hence to make a depth image, and then unwarp the image according to the depth image. Although it seems capable of unwarping any type of image distortions, how to map the points on the rough, noisy surface defined by the depth image to the points on the plane is still a problem. In [4], the scanned images of bound books are rectified by means of character segmentation. Characters in the shadow (along the binding line where the surface is curled) are located, estimating their orientations and rotating them to make them horizontal. Since the extent of bending is unknown by this method, it is not so accurate. In the resulting images, characters within the shadowy region still look narrower than characters in the rest region. In [5], a model of a combination of cylinder and plane is adopted to describe the surface of books, but how to estimate the parameters and make use of the model is unresolved, and it can only be applied to images scanned by the scanner. In [6], an applicable model is introduced to solve the more general case of document image warping. However, excessive approximation made the model inaccurate mathematically.

In this article, an analytically accurate cylindrical surface model (CSM) is proposed to represent and rectify the bound document image warping. This model can rectify a single image, in no need of image sequence or any stereographic device. In section 2 , the principle on how to use CSM to rectify the image is discussed. In section 3 , the detailed process of the rectification is described. Some experimental results indicating the performance of rectification and some application in real OCR system are presented in section 4 . The page is ended by section 5 with a summary on the proposed approach.

\section{The cylindrical surface model}

With a scanner, one may press the document on the glass so that the surface of the document is partially flat, whereas while taking photos, documents are often opened naturally, making the surface of the page close to a cylinder surface. The model described in [1] representing the surface by a combination of a part of circular cylinder and a semi-plane is no longer suit for the surface of the document. Therefore, a general cylinder surface of a non-circular directrix is adopted here to represent the surface of the document. Actually, the directrix may have 


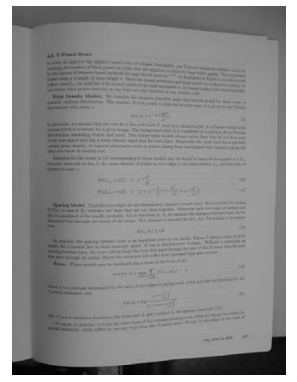

Figure 1. A warped image by bookbinding.

much more diverse shape.

According to [7], the image formation process of a lens system, such as the camera, can be approximately described by the perspective projection. In the case that the generatrix doesn't parallel the image plane, the mapping from the points of the generatrix to the points on the image plane can be considered as a nonlinear projective transformation, which is difficult to deal with. Therefore, in this article, the rectification problem is subject to the constraint that the generatrix of the cylinder parallels the image plane.

The geometry of the image formation process of the surface is demonstrated in figure 2. Assumed the generatrix of the cylinder parallels the image plane. On the image plane, take the point where the plane intersects with the optical axis as the origin, and select an $\mathrm{X}$ axis perpendicular to the generatrix, then a 2-D Cartesian coordinates frame is specified. By obey the same $\mathrm{X}$ and $\mathrm{Y}$ directions, and taking the optical axis as $\mathrm{Z}$ axis, one can get the 3-D coordinates frame shown in the figure 2 .

From the geometry of the image formation shown in figure 2, let's see how the warping is caused. Generally, the warping is the combined effect of two factors: (1) distance differences, which make farther pitch of the surfaces seem "compacted" compared with nearer ones, (2) foreshortening differences, which is related to the orientation of the surface. Foreshortening also makes the pitch of surface seem compacted. A skewer surface means a more significant effect of foreshortening. If the surface is perpendicular to the optical axis, there is no foreshortening. Both of these two effects exist in the CSM. Distance differences locally compact or dilate the image in both horizontal and vertical directions. While foreshortening only compact or dilate the image in horizontal direction.

Firstly, consider an arbitrarily selected directrix on the surface:

$$
l_{1}:\left\{\begin{array}{l}
y=y_{1} \\
z=D(x)
\end{array} .\right.
$$

Now try to get the equation of the projection of $l_{1}$ on the image plane, denoted by $L_{1}$. For the convenience, select the point $r\left(0, y_{1}, D(0)\right)$ from $l_{1}$ as the reference point,

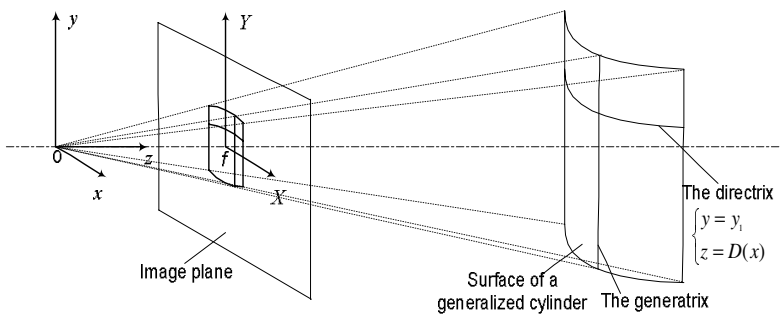

Figure 2. The process of image formation of the document surface (a cylindrical surface).

and its projection is $R\left(X_{0}, Y_{0}\right)=\left(0, \frac{f}{D(0)} y_{1}\right)$, where $f$ is

the focal distance. For any other point $P\left(\mathrm{x}, \mathrm{y}_{1}, \mathrm{D}(\mathrm{x})\right)$, assume that its image is $P^{\prime}(\mathrm{X}, \mathrm{Y})$. From the geometry, one may get

$$
X=\frac{f}{D(x)} x
$$

and

$$
Y=\frac{f}{D(x)} y_{1}
$$

Minus $\frac{f}{D(0)} y_{1}$ from both sides of (2), one can get

$$
Y-\frac{f}{D(0)} y_{1}=\frac{f y_{1}}{D(0) \cdot D(x)}[D(0)-D(x)]
$$

Notice that the depth difference in one directrix is often less than $1 \sim 2 \mathrm{~cm}$, while the average depth of the surface is mach larger, typically $50 \sim 100 \mathrm{~cm}$. In other words, $|D(x)-D(0)|<<D(0)$, where "<<" means "is far less than". Using this assumption, from (1) and (3), one can get:

$$
\begin{aligned}
& Y=\frac{f}{D(0)} y_{1}+\frac{f y_{1}}{D(0) \cdot\{[D(x)-D(0)]+D(0)\}}[D(0)-D(x)] \\
& \approx \frac{f}{D(0)} y_{1}+\frac{f y_{1}}{[D(0)]^{2}}[D(0)-D(x)] \\
& =\frac{2 f}{D(0)} y_{1}-\frac{f y_{1}}{[D(0)]^{2}} D(x)
\end{aligned}
$$

$=\frac{2 f}{D(0)} y_{1}-\frac{f y_{1}}{[D(0)]^{2}} D\left[\frac{D(x)}{f} X\right]$

Let $D^{*}(X)=\frac{f}{D(0)} D\left[\frac{D(0)}{f} X\right]$. Clearly, $D^{*}(x)$ is the curve $D(x)$ zoomed by $f / D(0)$ times, so they have identical shape. Notice that $\frac{D(x)}{D(0)}=\frac{f y_{1} / D(0)}{f y_{1} / D(x)}=\frac{Y_{0}}{Y}$, and $\frac{Y_{0}}{Y}$ is a function only related to $\mathrm{X}$ (see equation (1)). So denote $\rho(X)=\frac{Y_{0}}{Y}$. Practically, there may be some method to locate directrixes, so if given the coordinates $(X$, $Y$ ), one may find the corresponding $Y_{0}$ by tracing the projected image of the directrix that crosses $(X, Y)$, then 
estimate the value of $\rho(X)$ for each $X$. Then equation (4) can be convert to

$$
L_{1}: Y=\frac{y_{1}}{D(0)}\left[2 f-D^{*}(\rho(X) X)\right]
$$

Using the following coordinates transformation

$$
\left\{\begin{array}{l}
X^{\prime}=\rho(X) X \\
Y^{\prime}=Y
\end{array}\right.
$$

to transform the image from the $X-Y$ coordinates frame to a new $X^{\prime}-Y^{\prime}$ coordinates frame. So the resulting projected directrix $L_{1}$ ' satisfies

$$
L_{1}^{\prime}: Y^{\prime}=\frac{y_{1}}{D(0)}\left[2 f-D^{*}\left(X^{\prime}\right)\right]
$$

The transformation by equation (6) eliminates the warping caused by distance differences in $\mathrm{x}$-direction. So this process can be called by the $\mathbf{X}$-distance normalizing (XDN). More exited is that the projected image of any directrix in the 2-D image has a form similar to the function $D$, only different in scale or translation.

From equation (7), one can get the following result about the point in $L_{1}$ :

$$
2 f-D^{*}\left(X^{\prime}\right)=\frac{z^{*}}{y_{1}} Y^{\prime}=\frac{z^{*}}{h} \cdot \frac{h}{y_{1}} Y=\frac{z^{*}}{h} \cdot H \cdot \frac{Y}{Y_{0}} Y=\frac{z^{*}}{h} \cdot H \rho(X)
$$

where $h$ is the actual height of the scene captured in the image, $H$ is the height of the image, and $z^{*}=D(0)$ is the approximate distance of the surface. For the mapping $X^{\prime}=\rho(X) X$, denote its inverse by $X=\mathbf{X}\left(X^{\prime}\right)$. Then

$$
2 f-D^{*}\left(X^{\prime}\right)=\frac{z^{*}}{h} \cdot H \rho\left[\mathbf{X}\left(X^{\prime}\right)\right]
$$

One can calculate the waveform of $2 f-D^{*}\left(X^{\prime}\right)$ using equation (9).

Note $X^{\prime}=[f / D(0)] x$, where the constant scale factor is the same as that of $D^{*}$ to $D$. Then, by make two coordinate transformations in the $X^{\prime}$ and $Y^{\prime}$ directions, respectively, one can get the image rectified. The transformations are:

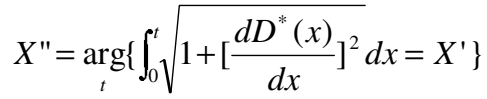

and

$$
Y^{\prime \prime}=\rho\left[\mathbf{X}\left(X^{\prime}\right)\right] Y
$$

In equation (10), the integration $\int_{0}^{t} \sqrt{1+\left[\frac{d D^{*}(x)}{d x}\right]^{2}} d x$ is the length of the curve $D^{*}(x)$ when $0 \leq x \leq t$. So this transformation means "flattening" the directrix. This process can be called by $\mathbf{X}$-foreshortening eliminating (XFE). Similar to the $\mathbf{X}$-distance normalizing, the transformation by equation (11) eliminates the warping of $\mathrm{y}$-direction caused by distance differences. This process can be called Y-distance normalizing (YDN). YDN maps all the points in a directrix to the same vertical ordinates in the $X$ '- $Y$ " coordinates frame.

\section{Rectifying the image using CSM}

With the CSM, the rectification can be performed. One can first locate the horizontal text lines in the image, and extract their baselines. Then use these lines to decide the left or right boundary lines of the column. A column is usually a rectangle region; hence one can specify the $\mathrm{Y}$ direction (and therefore the $\mathrm{X}$ direction) of figure 1 according to any of the two boundary lines. These baselines are taken as projected images of directrix to estimate the curve $\rho(X)$ and $2 f-D^{*}\left(X^{\prime}\right)$, and construct a flattened image by the approach in section 2 .

Figure 1 shows a document image of $1200 \times 1600$, the height of the real scene that seen from the image, $h \approx 28 \mathrm{~cm}$, and the distance from the lens to the surface of the document, $z^{*} \approx 59 \mathrm{~cm}$. The height of the image, $H=1600$ pixels. In the following of this section, let's take it as an example to see the key algorithms and processing in each steps of rectifying a warped image.

\subsection{Locating baselines of the text}

In most of the documents, the words in one text line are aligned along the baseline. If these baselines are located, they can quite accurately represent the directrixes.

Firstly, Niblack's algorithm [8] is adopted to threshold the image. Next the mathematical morphological method proposed by [9] is applied to get the baseline of the text. In the baseline image, a connected component searching operation is executed to get all of the base lines of each text line, hence to locate the directrixes. The searching strategy is that, select a start point from one end of a directrix, and search foreground points horizontally towards another end. In our experiments, each time from a foreground pixel, search for the next foreground pixel horizontally within 24 pixels and $\pm 12^{\circ}$.

The last text line of a paragraph usually stops half way. However, the searching process, if it is from the left to the right, sometimes does not stop, but traverses to an adjacent text line. To avoid this, the searching process is designed to run from the right to the left.

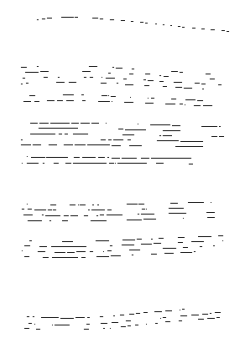

Figure 3. The base lines of text lines extracted from image in figure 1. 
The baselines of the text in figure 1 are shown in figure 5. The left and right column border-lines well indicate the Y-direction. One may achieve it by straight-line fitting.

\subsection{Estimating the curve $\rho(X)$}

By the processing in section 3.1, one can get several sets of pixels. Each set represents a projected image of a directrix., Use each set to predict a function values $\rho(X)$ for every $X$, then average all these functions to get a smooth curve of $\rho(X)$. With $\rho(X)$, the transformation of the coordinates frame in equation (6) can be achieved.

There may be no pixels in some set at certain coordinate $\mathrm{X}$. To solve this problem, before the above process, a interpolation is made using the neighboring pixels.

Figure 4 shows the resulting $\rho(X)$ for the image in Figure 1. Figure 5 shows the image in the transformed coordinates frame.

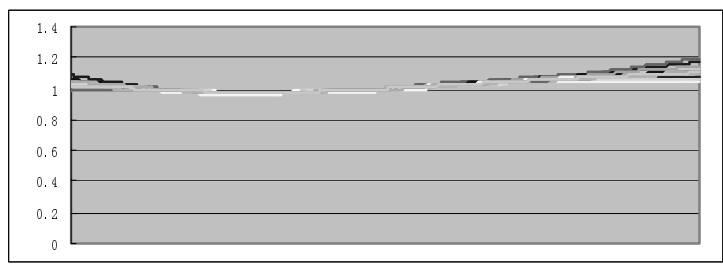

(a)

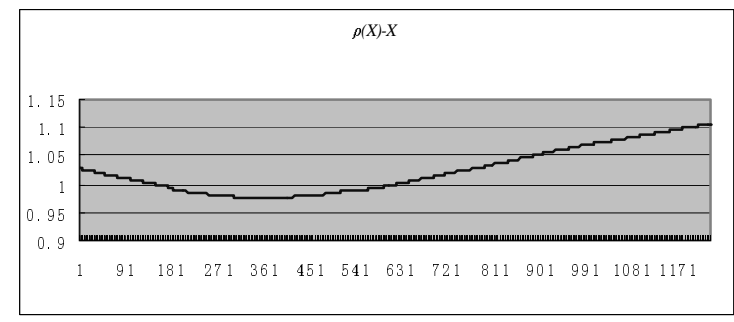

(b)

Figure 4. The curve $\rho(X)$ of the image in figure 1. (a) $\rho(X)$ calculated by all directrixes. (b) The averaged $\rho(X)$ of all the curves from (a).

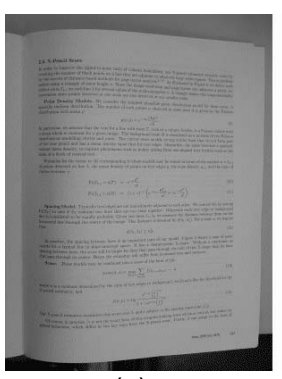

(a)

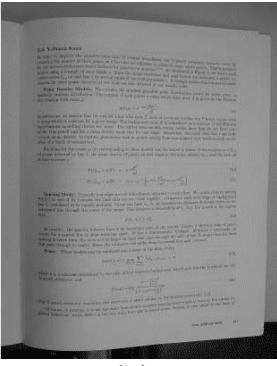

(b)
Figure 5. XND using $\rho(X)$. (a) The original image. (b) The resulting image, visually slightly wider than the original one.
Notice that in Figure 4(a), the waveforms at the two ends of horizontal axis are diverse. The reason is that the waveforms of these intervals are not estimated by real data, but by interpolated data. The valid $X$ value of text region is between 130 and 1030 pixels. Within this interval, the variance of all the waveforms is slight, hence to ensure an accurate estimation of $\rho(X)$.

\subsection{Estimating the curve $2 f-D^{*}\left(X^{\prime}\right)$}

Like calculating the curve $\rho(X)$, all directrixes are used to estimate the curve $2 f-D^{*}\left(X^{\prime}\right)$. Figure 6 shows the resulting curve $2 f-D^{*}\left(X^{\prime}\right)$. The valid $X^{\prime}$ value of text region is between 144 and 1080 pixels.

First, a 5-point averaging operator is applied to smooth the curve $2 f-D^{*}\left(X^{\prime}\right)$, and then the first order difference $\left[2 f-D^{*}\left(X^{\prime}+1\right)\right]-\left[2 f-D^{*}\left(X^{\prime}\right)\right]$ is taken to approximate the derivative $\frac{d\left[2 f-D^{*}\left(X^{\prime}\right)\right]}{d x}$. An example of smoothing is shown in figure 7. You will see the first order difference

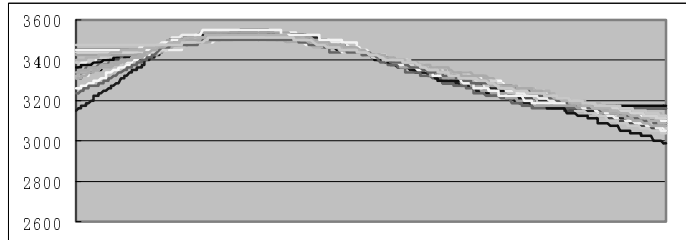

(a)

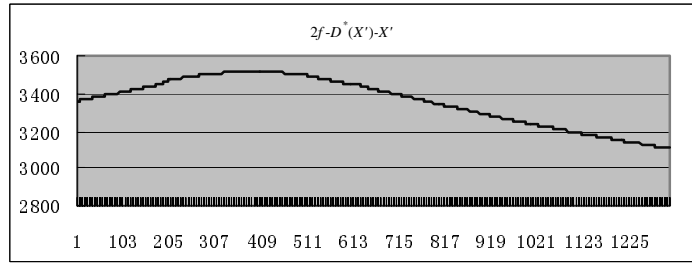

(b)

Figure 6 . The curve $2 f-D^{*}\left(X^{\prime}\right)$ of the image in Figure 1. (a) $2 f-D^{*}\left(X^{\prime}\right)$ calculated by all directrixes. (b) Averaged $2 f-D^{*}\left(X^{\prime}\right)$ of all the curves in (a).

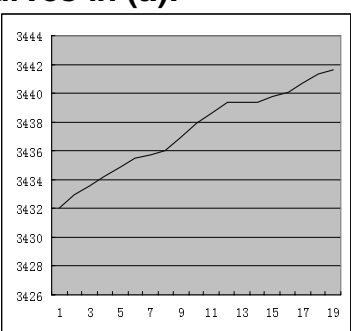

(a)

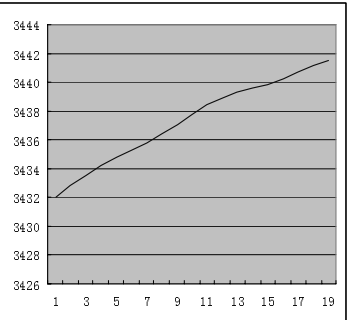

(b)
Figure 7. Curve smoothing. (a) A fraction of row curve. (b) The smoothed curve (by 5-point averaging ). 


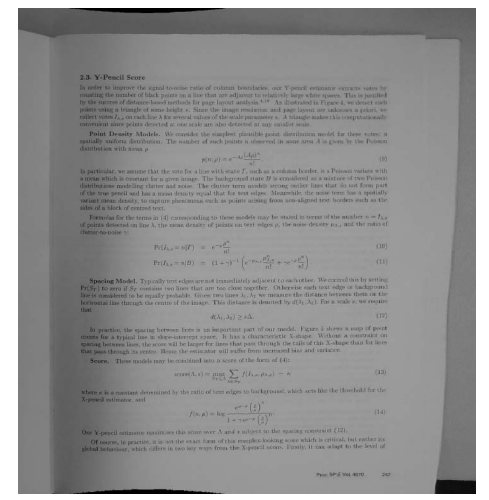

Figure 8. The rectified image of figure 1.

distribution distribution distribution
(a) 89pix long
(b) 100pix long
(c) 86 pix long

\section{distribution distribution distribution distribution distribution distribution}
(a') 100pix long
(b') 100pix long
(c') 99pix long

Figure 9. (a)-(c) Image patches of the word "distribution" extracted from the original image. (a')-(c') Corresponding image patches from the rectified image.

is quite close to the derivative in the smoothed curve.

Next, it is time to use the transformation by equations (10) and (11) to get the rectified image.

\section{Analyses and discussions on experimental results}

\subsection{The final result}

The rectified image of the one in Figure 1 is shown in figure 8 . Notice that the text lines are quite straight and the widths of characters are uniform. To see this clearly, we select the image pitches of the word "distribution" in three different locations of the image, and compare them between the original image and the rectified one, as shown in figure 9. Binarised images and lengths of each word (in pixels) are also given in figure 9 . These words get almost the same orientations and lengths after rectifying.

We have tried our method on around forty sample images of different languages (including English and Chinese) and different resolution ranges from about 90dpi to 220dpi. Some of the results are shown in figure 10. In our experiment, most of the images get rectified, except a few on which our program cannot find the Y-direction accurately. In these images, too many indented lines or lines that are the end of a paragraph in a single page result in the error of line-fitting. When we use manually estimated Y-directions instead, each time the rectifying algorithm works well. We are now investigating methods

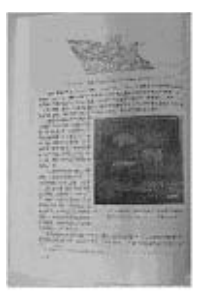

(a)

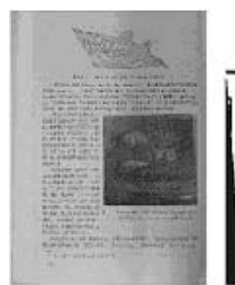

(a')

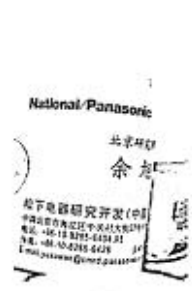

(b)

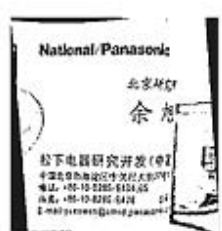

(b')

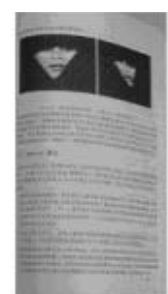

(c)

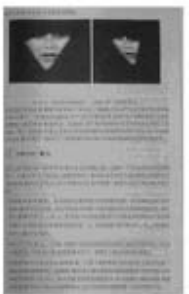

(c')
Figure 10. Some of the results: $(a)-(c)$ are the original images, and $\left(a^{\prime}\right)-\left(c^{\prime}\right)$ are the corresponding results.

on a better estimation of Y-direction.

\subsection{Applied to the pre-processing of OCR}

To see how our method can be used in some applications, we compare OCR tests between the original and the rectified images. We take arbitrarily some samples in English and some in Chinese, and use ScanSoft Omnipage Pro 11.0 and TH-OCR 2000 to make the OCR test of English and Chinese samples, respectively. Before OCR, the unrectified and rectified images are zoomed in two times in both the width and the height by bilinear interpolation to achieve the proper resolution (200 to 300 dpi) required by the OCR system, then they are binarised using Niblack's algorithm of the same window size (39 by 39). Then use OCR software to recognize the document images in the "automatic" mode of OCR software, i.e., automatically analyzing the layout, segmenting words, and recognizing them without any human interference. A group of results of automatic layout analysis are shown in figure 11, where the one in the left shows the layout of the original image, and the one in the right shows the layout of the rectified version of the left one. Without the process of rectification, the layout analysis can hardly produce a correct result (see figure 11(a)), and the character segmentation is not reliable, producing disagreeable segmentation errors. A typical segmentation error is shown in figure 12 , where two adjacent text lines cannot be split apart. This is due to the existence of warp in the image. While in the rectified image, the horizontal text lines are straight. The recognition performances are listed in table $1 \& 2$. As can be seen from these two tables, the rectification process before OCR can effectively reduce recognition errors. 

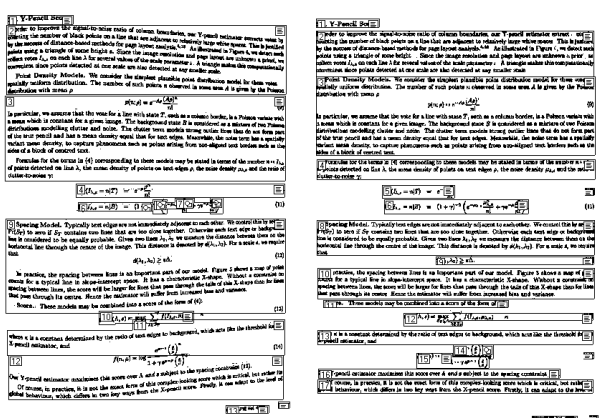

Figure 11. The layout analysis result of the original image and the rectified image using OmniPage. (a): the layout of the original image; (b): the Layout of the rectified image.

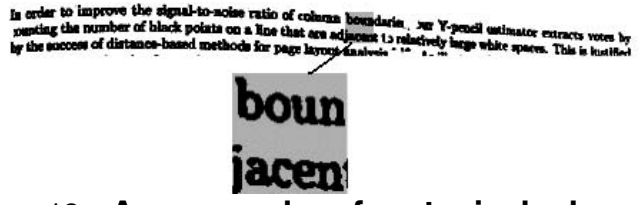

Figure 12. An example of a typical character segmentation error due to the existence of skew in the original image. The shadow region is an incorrect word region acquired by the segmentation.

\subsection{Cost of computing time}

The time cost is mainly in the processes of finding the base lines and producing the new image. The estimation of $\rho(X)$ and $2 f-D^{*}\left(X^{\prime}\right)$ do not spent much, because relatively fewer feature points are involved in the estimations of curves, compared with the former processes.

Generally, it takes around 3 seconds to rectify a $1200 \times 1600$ image (CPU: Intel $1.7 \mathrm{GHz}$ ). This is usually much faster than the OCR process.

\section{Summary}

In this article, a cylindrical surface model is proposed to describe the relationship between the surface of the bound document and its image formed on the image plane. Through some image analysis and processing operations, our experiment results showed that it is possible to rectify the images using the model. This method is then applied to the pre-processing of OCR, making remarkable improvement in the recognition quality.

The proposed approach required that the generatrixes of the cylinder parallel the image plane. As for the photos taken freely from any angle of view, the process of rectification may be much harder. We will seek the solution of this problem in further investigations.
Table 1. The recognition performances of the original images and the rectified images (of English text, using OmniPage).

\begin{tabular}{|l|c|c|c|l|}
\hline Images & $\begin{array}{l}\text { Number } \\
\text { of } \\
\text { English } \\
\text { words }\end{array}$ & $\begin{array}{l}\text { Correctly } \\
\text { recognized } \\
\text { English } \\
\text { words }\end{array}$ & $\begin{array}{l}\text { Recognition } \\
\text { rate }\end{array}$ & $\begin{array}{l}\text { Error rate } \\
(\%)\end{array}$ \\
\hline $\begin{array}{l}\text { Not } \\
\text { rectified }\end{array}$ & 1962 & 1808 & 92.15 & 7.85 \\
\hline Rectified & 1962 & 1897 & 96.69 & 3.31 \\
\hline
\end{tabular}

Table 2. The recognition performances of the original images and the rectified images (of Chinese text, using TH-OCR).

\begin{tabular}{|l|c|c|c|c|}
\hline Images & $\begin{array}{l}\text { Number } \\
\text { of } \\
\text { Chinese } \\
\text { characterscharacters }\end{array}$ & $\begin{array}{l}\text { Correctly } \\
\text { recognized } \\
\text { Chinese }\end{array}$ & $\begin{array}{l}\text { Recognition } \\
\text { rat }\end{array}$ & $\begin{array}{l}\text { Error } \\
\text { rate (\%) }\end{array}$ \\
\hline Not & & & 45.7 & 54.3 \\
\hline rectified & 1675 & 766 & 9.5 & 9.5 \\
\hline Rectified & 1675 & 1471 & 90.5 & \\
\hline
\end{tabular}

\section{Acknowledgments}

This work was supported by 863 Hi-tech Plan (project 2001AA114081) \& National Natural Science Foundation of China (project 60241005).

\section{References}

[1] A. Doncescu, A. Bouju, V. Quillet, "Former books digital processing: image warping," in Proc. Workshop of Document Image Analysis, 5-9, 1997.

[2] D. B. Smythe, "A Two-Pass Mesh Warping Algorithm for Object Transformation and Image Interpolation", ILM Technical Memo \#1030, Computer Graphics Department, Lucasfilm Ltd., 1990.

[3] M. S. Brown, W. B. Seales, "Document restoration using 3D shape: a general deskewing algorithm for arbitrarily warped documents", in Proc. International Conference on Computer Vision, July 2001.

[4] Z. Zhang, C. L. Tan, "Restoration of images scanned from thick bound documents", in Proc. 6th International Conference on Document Analysis and Recognition, 2001.

[5] T. Kanungo, R. Haralick, I. Philips, "Global and local document degradation models," in Proc. 2nd International Conference on Document Analysis and Recognition, 1993.

[6] H. Cao, X. Ding, C. Liu, "Rectifying the Bound Document Image Captured by the Camera: A Model Based Approach" in Proc. 7th International Conference on Document Analysis and Recognition, 2003.

[7] S. Russell and P. Norvig, Artificial Intelligence: A Modern Approach, 1st Edition, pp. 725-727 Prentice Hall, 1995.

[8] W. Niblack, An Introduction to Digital Image Processing, Prentice Hall, Englewood Cliffs, 1986.

[9] A.K. Das, B. Chanda, "A fast algorithm for skew detection of document images using morphology," in IJDAR 2001, vol. 4, $109-114$. 